\title{
Determinação de íons amônio em eletrodos modificados com polipirrol
}

\section{Ammonium ions determination with polypyrrole modified electrode}

\author{
Luciana T. S. Mendes ${ }^{1}$, Luiz Henrique Dall’Antonia ${ }^{2}$
}

Resumo

O trabalho teve como objetivo a preparação de filmes de polipirrol (PPy) depositado sobre superfícies de carbono vítreo, níquel e ITO (óxido de estanho dopado com Índio) em plástico tipo PET, a fim de estudar suas propriedades em relação à detecção de amônia. Os filmes de polipirrol dopados com dodecilbenzenosulfonato (DBSA) foram formados sobre os eletrodos, a + 0,70 V vs. Ag/AgCl, a partir de uma solução contendo o monômero pirrol e o sal anfifílico sendo que os filmes depositados sobre carbono vitreo apresentaram melhor performance. Voltametrias cíclicas, entre - 1,50 até +0,5 V vs. Ag/ $\mathrm{AgCl}$, foram repetidas acrescentando diferentes concentrações de $\mathrm{NH}_{4} \mathrm{Cl}$, a fim de observar o comportamento do filme como um possível detector de íons $\mathrm{NH}_{4}^{+}$. A corrente do pico de oxidação em função da concentração de amônia para esses filmes varia com a quantidade de $\mathrm{NH}_{4} \mathrm{Cl}$. Observa-se uma região linear, na faixa de 0 a $80 \mathrm{mM}$, com uma sensibilidade $\left(\mathrm{S}_{\mathrm{ppy}}\right)$ aproximadamente igual a 4,2 $\mathrm{mA} \mathrm{mM}$ ${ }^{1} \mathrm{~cm}^{-2}$, demonstrando a eficácia dos eletrodos como sensores de íons amônio. A quantidade de polímero depositada, controlada pelo tempo de crescimento, não influi na sensibilidade do sensor. O eletrodo modificado foi usado para determinar amônio em águas subterrâneas.

Palavras-chave: Amônio. Sensor. Polipirrol.

\begin{abstract}
The present work relates the preparation of polypyrrole films (PPy) deposited on surfaces of glass carbon, nickel and ITO (tin oxide doped with indium) on PET plastic, in order to study the ammonium detection. The popypyrrole films were polymerized with dodecylbenzenesulfonate (DBSA) on the electrodes, at $+0,70 \mathrm{~V}$ vs. $\mathrm{Ag} / \mathrm{AgCl}$, based on a solution containing the pyrrole monomer and the amphiphilic salt. Films deposited on glass carbon presented better performance. Cyclic voltammetries, between $-1,50$ to $+0,5 \mathrm{~V}$ vs. $\mathrm{Ag} / \mathrm{AgCl}$, were repeated adding different concentrations of $\mathrm{NH}_{4} \mathrm{Cl}$, in order to observe the behavior of the film as a possible detector of ions NH4+. The peak current for oxidation varies with the concentration of ammonium. A linear region can be observed in the band of 0 to $80 \mathrm{mM}$, with a sensibility $\left(\mathrm{S}_{\mathrm{ppy}}\right)$ approximately similar to $4,2 \mathrm{~mA} \mathrm{mM}^{-1} \mathrm{~cm}^{-2}$, showing the efficacy of the electrodes as sensors of ammonium ions. The amount of deposited polymer, controlled by the time of growth, does not influence on the sensor sensibility. The modified electrode was used to determine ammonium in grounded waters.
\end{abstract}

Key words: Ammonium. Sensor. Polypyrrole.

1 Bacharel em Química - Laboratório de Eletroquímica e Materiais/Depto. Química/CCE/UEL.

2 Professor Adjunto - Laboratório de Eletroquímica e Materiais/Depto. Química/CCE/UEL. E-mail: luizh@uel.br 


\section{Introdução}

A determinação da concentração de amônia sempre foi assunto de grande interesse, por ser muito importante para o diagnóstico de algumas doenças, como a insuficiência renal e hepática e o diabetes. Outro fator bastante importante na determinação de amônia reside no fato de que a presença desta em água potável e alimentos indica a decomposição de materiais orgânicos que podem ser prejudiciais à saúde humana. Por outro lado, a determinação da concentração de amônia em solos onde sais de amônia é utilizada como fertilizantes é também importante, devido ao fato que um excesso de amônia altera a acidez do solo, quebrando o ciclo de nutrição e o balanço ecológico (WYERS; OTJES; SLAMINA, 1993). Baseados em necessidades como citadas acima, pesquisadores tem desprendido esforços no estudo de novos sensores para amônia. Um sensor de amônia baseado em polipirrol foi uma das primeiras realizações práticas da utilização de polímeros condutores como sensor (NYLANDER; ARMGARTH; LUNDSTROM, 1983). Porém sua sensibilidade era muito baixa e sua resposta completamente irreversível. Investigações mais extensivas foram realizadas por Miasik, Hoper e Tofield (1986), cujos experimentos revelaram que uma camada de polipirrol, depositada em um eletrodo de trabalho, poderia ser utilizada na detecção de amônia e dióxido de nitrogênio. Sua condutância decrescia (aumentava) em um ambiente com amônia (dióxido de nitrogênio). A explicação para esse comportamento foi baseada nos conceitos de estado sólido tradicionais. A condução no filme de polipirrol é do tipo $\mathrm{p}$, assim gases que funcionam como receptores de elétrons, no caso o dióxido de nitrogênio, atraem os elétrons das moléculas do polímero, aumentando a condutância, e o contrário acontece com a amônia que funciona como doador de elétrons. Os sensores de polipirrol demonstraram uma boa reversibilidade de resposta para amônia na região de $100-10000$ ppm, mas a dependência da resposta com a concentração não foi essencialmente linear, além de que para baixas concentrações o tempo de resposta era de algumas horas. Outros estudos foram realizados para polipirrol com camada sensitiva, porém os sensores apresentaram baixa sensibilidade e uma resposta não reversível (BLANC et al., 1990; GUSTAFSSON; LUNDSTRÖM, 1987). Lubentsov e colaboradores realizaram experimentos para investigar o efeito de amônia e água em filmes de polianilina (PANI) medindo as mudanças na condutividade e a dependência da temperatura de desorção (LUBENTSOV; TIMOFEEVA; KHIDEKEL, 1991). Comparado ao polipirrol, a PANI apresenta uma sensibilidade muito maior para a amônia, bem como tempos de respostas mais curtos (1-2 minutos). Um outro sensor baseado em PANI dopado com ânions contendo níquel foi construído por Krutovertsev e colaboradores, e nele se observou uma maior sensibilidade ao gás amônia (1-10000 ppm) atribuída aos ânions aditivados ao filme (KRUTOVERTSEV et al., 1992). Outros trabalhos também descreveram o uso de filmes de PANI como sensores de amônia (WNEK, 1986), porém esses sensores também apresentaram uma resposta não reprodutível, além de ocorrem problemas tecnológicos durante a deposição da camada de polímero.

Sensores amperométricos de amônia baseados em polipirrois e polipirrois substituídos

O primeiro trabalho a respeito ao uso de polipirrol como eletrodo modificado e a potencialidade para a detecção de amônia foi elaborado por Kanazawa e colaboradores (KANAZAWA et al., 1979), e os resultados revelaram que a exposição de um filme de polipirrol em face da amônia provoca a diminuição da condutividade, à temperatura ambiente, em um fator de 10. Porém estes autores relataram que a amônia interage fracamente com o PPy e pode ser facilmente removida, de modo que se restaura a condutividade do polímero ao seu estado original.

A sensibilidade da resistência dos filmes de polipirrol em relação à amônia, foram demonstradas por Nylander e colaboradores (NYLANDER; ARMGARTH; LUNDSTRÖM, 1983). Gustafsson e colaboradores (GUSTAFSSON; LUNDSTRÖM, 1987; GUSTAFSSON et al., 1989) demonstraram 
que, em baixas concentrações de amônia, o aumento provocado na resistência é reversível, enquanto concentrações acima de $1 \mathrm{~atm}$, ou na presença de água leva a um aumento irreversível da resistência. $\mathrm{O}$ aumento da resistência foi atribuído ao ataque nucleofílico da amônia ou hidróxido no polímero provocando a uma perda na conjugação e ainda uma abertura do anel. Filmes preparados eletroquimicamente apresentaram uma detecção da ordem de 0,01 \% em amônia (MIASIK et al., 1987). Também um filme muito fino de ppy depositado sobre um substrato não condutor pode ter um uso como um quimiresistor, para a detecção de amônia e antrazina (RATCLIFFE, 1990).

Mudanças reversas na resistência foram observadas em experimentos d. c. de condutividade realizados em filmes de polipirrol dopados com hexacianoferrato, nas quais os resultados foram obtidos para altas concentrações de amônia (BLANC et al., 1990). Foi concluído através deste experimento que a amônia atua com um desdopante, causando o decréscimo a condutividade.

Estudos envolvendo experimentos da mudança resistência de PPy e massa na presença de alguns gases e vapores, incluindo amônia, levaram a conclusão de que o mecanismo de resposta da sensibilidade do PPy é devido a uma resposta mista envolvendo efeitos eletrônicos e físicos (SLATER et al., 1992). Mudanças de massa foram utilizadas para desenhar um sensor de gás piezoelétrico com um filme eletroquimicamente depositado de PPy na superfície de um quartzo pisoelétrico (SLATER et al., 1992; VIGMOND et al., 1992).

Trojanowicz e colaboradores demonstraram que eletrodos de platina modificados com um filme de PPy podem ser utilizados como sensores amperométricos para amônia em soluções aquosas (TROJANOWICZ et al., 1996). E mais recentemente, Lähdesmäki e colaboradores (LÄHDESMÄKI et al., 2000) apresentaram resultados para filmes de PPy e especulações quanto ao mecanismo de detecção de amônia, sem ao certo elucidar os fatos.
Porém o mecanismo de detecção de amônia pelo filme polimérico ainda não tem sido muito bem explicado e algumas controvérsias existem, com respeito ao envenenamento do eletrodo. Um mecanismo proposto por Gustafson e colaboradores (GUSTAFSSON; LUNDSTRÖM, 1987; GUSTAFSSON et al., 1989), explicou o mecanismo por meio de uma reação reversível da desprotonação do polímero com amônia nucleofílica, de acordo com a equação 1 .

$$
\mathrm{PPyH}^{+} \mathrm{Cl}^{-}+\mathrm{NH}_{3} \rightleftarrows \mathrm{PPy}^{0}+\mathrm{NH}_{4} \mathrm{Cl}^{-}
$$

Porém a perda de eficiência na detecção e a irreversibilidade do processo de detecção para altas concentrações de amônia, não pôde ser explicado pela reação acima. Alguns pesquisadores atribuem essa ineficiência ao ataque nucleofílico da amônia ou hidróxido no polímero, esse provoca uma perda na conjugação e ainda uma abertura do anel (GUSTAFSSON; LUNDSTRÖM, 1987; GUSTAFSSON et al., 1989; TROJANOWICZ et al., 1996; LÄHDESMÄKI et al., 2000). Entretanto, foi demonstrado que mesmo após uma exposição por longo período de tempo, o filme polimérico ainda pode ser regenerado pela ciclagem do mesmo em uma solução fortemente ácida (HEIDUSCHKA et al., 1997), o que, de certa forma, invalida os mecanismo de ruptura do anel prevendo uma perda irrecuperável do eletrodo.

Recentes estudos (DALL'ANTONIA et al., 2002; VIDOTTI et al., 2003; VIDOTI et al., 2004) demonstraram que filmes de polipirrol dopados com DBSA mostraram-se muito promissores para serem empregados na construção de sensores amperométricos para determinação de quantidades de amônia, uma vez que a sensibilidade à amônia estende-se por uma região $(0$ a $1 \mathrm{mM})$ até quatro vezes maior do que filmes dopados com íons cloreto e apresentam uma resposta de corrente bastante acentuada. Experimentos eletrogravimétricos demonstraram que durante o processo de determinação amperométrica de amônia, há o 
intercâmbio de cátions e ânions da solução tampão para o interior do filme, sendo este transporte depende do dopante utilizado na preparação do filme polimérico.

O mecanismo de detecção da amônia por filmes poliméricos de PPy pode ser verificado por meio de espectroscopia Raman, no qual a diminuição da banda referente a deformação no plano N-H, é um indicio do mecanismo para a detecção de amônia no estado gasoso, equação 1, proposto por Gustafsson e colaboradores (GUSTAFSSON; LUNDSTRÖM, 1987; GUSTAFSSON et al., 1989). Porém nenhuma evidência de que uma destruição da cadeia polimérica, com consequente ruptura do anel pirrólico, pode ser observado por espectroscopia Raman, de modo que para filmes expostos há uma elevada concentração de amônia .

Baseados nos resultados de espectroscopia Raman e nos resultados eletrogravimétricos (DALL'ANTONIA et al., 2002), foi proposto que o envenenamento do eletrodo, observado pela perda de resposta, quando este era submetido a altas concentrações de analito, deve-se ao fato de uma perda de eletroatividade gerada pela inserção e retenção de anions provenientes da solução tampão e não pela destruição da cadeia polimérica. Essa hipótese é corroborada pelos resultados obtidos por Göpel e colaboradores (HEIDUSCHKA et al., 1997) para a regeneração de filmes de PPy após longos tempos de exposição a altas concentrações de amônia pela simples oxidação e redução do filme polimérico em uma solução de ácido perclórico. Com isso, proporciona-se uma troca dos ânions incorporados durante a exposição do filme à amônia.

O presente trabalho teve como objetivo a preparação de filmes de polipirrol (PPy) depositados sobre superfícies de níquel, ITO e carbono vítreo, de modo que fosse dado a investigar as propriedades eletrônicas dos mesmos em relação à determinação de íons amônio. Como aplicação dos eletrodos modificados com melhor desempenho foi medida a concentração de amônio em águas subterrâneas coletadas no aterro sanitário de Londrina.

\section{Experimental}

\section{Preparo das soluções}

Foram preparadas solução de pirrol $(0,05 \mathrm{M})$ dopado com $\mathrm{KCl}(0,5 \mathrm{M})$ e solução de pirrol $(0,05$ M) dopado com o surfactante DBSA (0,05 M). Para a primeira solução, diluiu-se em um balão volumétrico de $25 \mathrm{~mL}, 0,0865 \mathrm{~mL}$ de pirrol (purificado por destilação e conservado sob refrigeração) e $0,932 \mathrm{~g}$ de $\mathrm{KCl}$ em água destilada com o auxílio de ultrasom. Para a segunda solução, foi realizado o mesmo procedimento utilizando $0,0865 \mathrm{~mL}$ de pirrol e 0,436 $\mathrm{g}$ de DBSA. Foram preparadas também soluções de $\mathrm{KCl}(0,5 \mathrm{M})$ e $\mathrm{NH}_{4} \mathrm{Cl}(5 \mathrm{M})$.

\section{Preparação dos filmes poliméricos}

Os filmes de PPy foram preparados a partir de soluções de PPy dopados com $\mathrm{KCl}$ e PPy dopados com DBSA, aplicando-se um potencial de $0,7 \mathrm{~V}$ e $0,75 \mathrm{~V}$ com o potenciostato por diferentes tempos (30s, 60s, 120s, 360s, 720s), a fim de se obter várias espessuras de filme sobre os seguintes eletrodos de trabalho: Níquel, ITO plástico e Carbono Vítreo.

\section{Voltametria cíclica}

Após lavar com água destilada o eletrodo com o filme depositado, realizou-se a voltametria cíclica em $\mathrm{KCl} 0,5 \mathrm{M}$ a uma velocidade de $20 \mathrm{mV} / \mathrm{s}$ em um intervalo de potencial de $-1,5 \mathrm{~V}$ a $0,5 \mathrm{~V}$. Foram registrados 5 ciclos para cada espessura de filme em cada um dos eletrodos.

A partir das análises dos voltamogramas cíclicos, foram escolhidos o eletrodo e a solução que obtiveram os melhores resultados. Realizou-se novamente a voltametria cíclica utilizando um volume fixo de $\mathrm{KCl}$ e variando o volume de $\mathrm{NH}_{4} \mathrm{Cl}$, a fim de observar o comportamento do filme como um potencial detector de íons $\mathrm{NH}_{4}^{+}$.

\section{Curva Analítica}

Foram realizadas diversas voltametrias cíclicas, variando-se a concentração de $\mathrm{NH}_{4} \mathrm{Cl}(0,05$ a $1 \mathrm{mg} / \mathrm{L})$, uma vez que o valor máximo permitido de amônia em água de distribuição é de $0.08 \mathrm{mg} / \mathrm{L}$. Para cada 
concentração, anota-se o valor da corrente de pico que é utilizada como curva analítica.

Foram realizadas as voltametrias cíclicas utilizando as amostras do lixão, e anotado as suas correntes de pico. Depois se relacionou a corrente de pico encontrada nas amostras com a concentração de nitrogênio baseando-se na curva analítica. Utilizando a equação da reta da curva de calibração, foram encontradas as concentrações de nitrogênio amoniacal nas amostras.

\section{Coleta de amostras}

Uma vez que a água parada no poço pode não ser representativa da qualidade da água local, faz se necessário à remoção desta, de tal forma, que a água da formação a substitua. É importante utilizar uma técnica adequada de esgotamento de poços, para que não haja comprometimento da qualidade da água. Após o esgotamento e assim que o poço se recuperar suficientemente, é feita à coleta da amostra. Os primeiros parâmetros que devem ser avaliados são $\mathrm{pH}$, temperatura e condutividade, e devem ser medidos, se possível, no local.

A fim de reduzir as possibilidades de contaminação da amostra, utiliza-se um amostrador específico (beiler) para cada poço. Os frascos também devem ser limpos conforme o parâmetro de interesse, mas geralmente é lavado com detergente (preferencialmente não fosfatado) e água de torneira e enxaguados com água destilada. Pode-se empregar também tratamento com ácido crômico para remoção de depósitos orgânicos.

\section{Resultados e Discussão}

Trabalhos publicados relatam que a síntese de PPy na presença de volumosos surfactantes anfifílicos resulta na formação de filmes que melhoram as propriedades eletroquímicas e mecânicas (TORRESI et al., 1995). Os voltamogramas cíclicos para dois filmes de polipirrol (um deles dopado com cloreto, e o outro com DBSA, Figura 1, em solução 1,0 M de $\mathrm{KCl})$ demonstram uma mudança significativa que pode ser observada no perfil dos dois voltamogramas, indicando que o surfactante tem uma significante influência na resposta eletroquímica dos filmes de PPy. Os picos observados nos voltamogramas sugerem um processo redox complicado para o PPy/ DBS. Como observado por De Paoli e colaboradores, filmes de PPy dopados com ânions anfifílicos apresentam um processo redox dependente do eletrólito utilizado. Contrariamente com o observado para filmes de PPy dopados com ânions inorgânicos de baixo peso molecular, os ânions anfifílicos não difundem para a solução. Este fato pode ser parcialmente atribuído para o tamanho e compatibilidade do final polar com a matriz carregada e do final apolar com a matriz neutra (reduzida) (TORRESI et al., 1995).

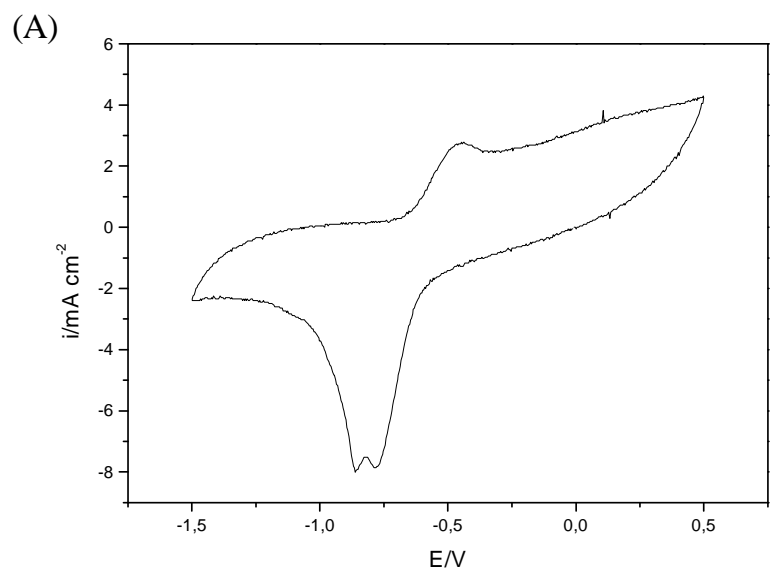

(B)

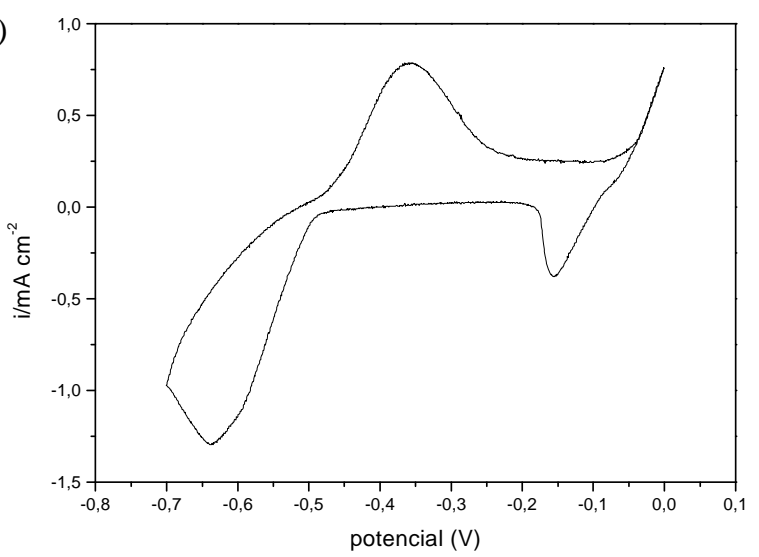

Figura 1. Voltamogramas Cíclicos para filme de polipirrol dopado com (A) DBSA; e (B) Cloreto, sobre eletrodo de carbono vítreo em solução aquosa de $\mathrm{KCl} 1,0 \mathrm{M}$. Velocidade de varredura igual a $20 \mathrm{mV} / \mathrm{s}$. 
O processo de redução do PPy/DBSA produz um polímero neutro, entretanto, a presença de ânions DBSA remanescentes conduz a inserção de cátions de maneira a equilibrar a carga. Porém além da dependência observada para a diferente natureza do cátion, também uma grande dependência quanto a natureza do ânion também já foi descrita na literatura (TORRESI et al., 1995). Dessa maneira, deduz se que o mecanismo do processo redox deve envolver tanto a presença do cátion como a do ânion presente no eletrólito. O processo redox envolvendo cátions e ânions pode ser representado por:

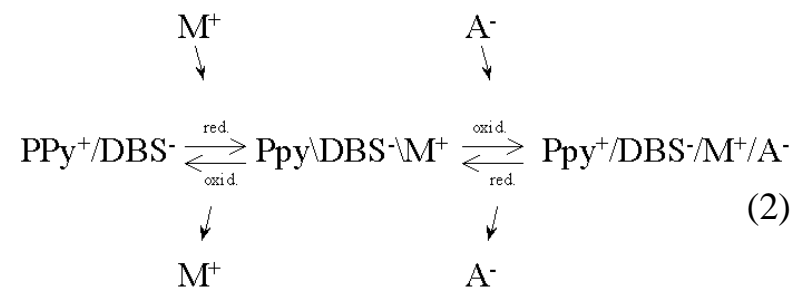

De acordo com este mecanismo, não há uma dessorção de DBSA quando o polímero é reduzido nos primeiros ciclos, mas a inserção de cátions $\mathrm{M}^{+}$ do eletrólito (etapa 1) formando um aducto PPy/DBS$/ \mathrm{M}^{+}$. Entretanto, quando o polímero é reoxidado deve ocorrer a desorção de $\mathrm{M}^{+}$(etapa 1) ou a inserção de ânions $\mathrm{X}^{-}$provenientes do eletrólito (etapa 2) produzindo um aducto $\mathrm{PPy}^{+} / \mathrm{DBS}^{-} / \mathrm{M}^{+} / \mathrm{X}^{-}$.

Quando filmes de PPy são dopados com ânions inorgânicos de baixo peso molecular, o mecanismo redox envolve a compensação de cargas pela inserção de ânions na matriz polimérica compensando a carga positiva resultante da oxidação. Assim, o processo redox pode ser representado por:

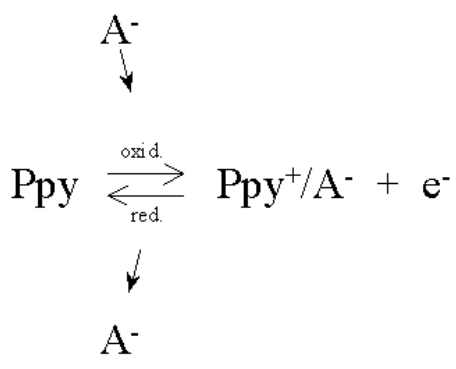

Torna-se evidente que a incorporanção do DBSA na matriz de polipirrol provocará mudanças na resposta do eletrodo quando utilizado na determinação quantitativa de amônia. Os filmes formados sobre eletrodo de Carbono Vítreo em solução de pirrol e DBSA, foram os que apresentaram melhor desempenho. Os picos que aparecem nos voltamogramas cíclicos indicam que uma reação com transferência de elétrons está ocorrendo. Portanto, quanto maior a altura desse pico, maior a quantidade de substância que está sofrendo oxidação ou redução.

$\mathrm{Na}$ Figura 2, são apresentados os resultados da corrente gerada em função da concentração de amônia para filmes de polipirrol dopados com DBSA em carbono vítreo. É verificado que o potencial de pico anódico não sofre bruscas variações com a quantidade de cloreto de amônio adicionado, assim os valores de corrente de pico anódico foram obtidos para um valor de potencial igual a $500 \mathrm{mV}$. Observase uma região linear, na faixa de 0 a $80 \mathrm{mM}$, com uma sensibilidade $\left(\mathrm{S}_{\mathrm{ppy}}\right)$ aproximadamente igual a 4,2 $\mathrm{mA} \mathrm{mM}^{-1} \mathrm{~cm}^{-2}$.

(A)

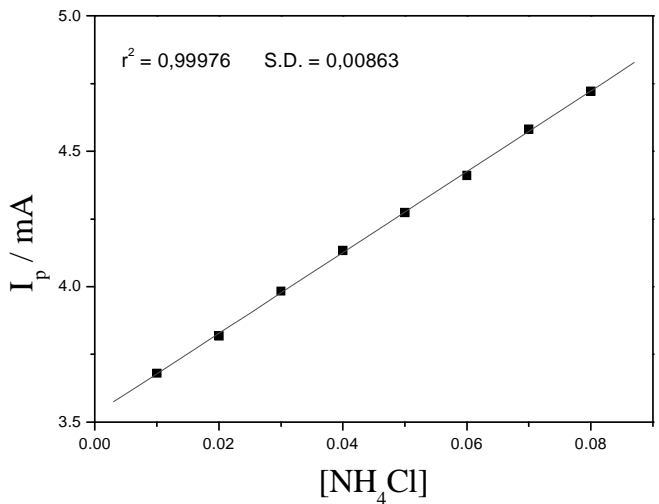

(B)

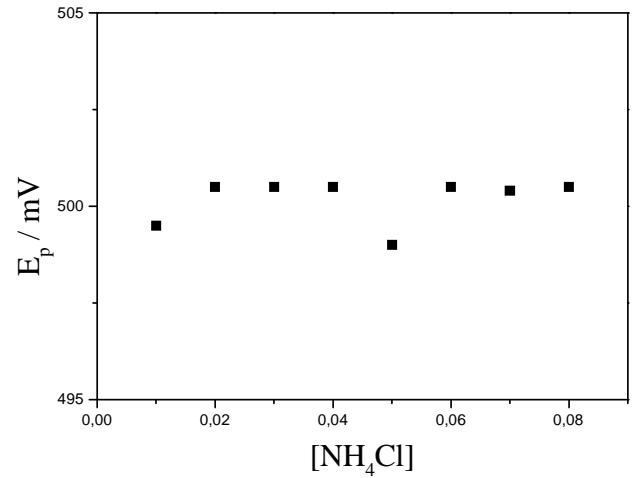

Figura 2. Dependência da resposta do filme de polipirrol dopado com íons DBSA sobre a concentração de íons amônio em KCl $1 \mathrm{M}$. 
A amônia é um tóxico bastante restritivo à vida dos peixes, e muitas espécies não suportam concentrações acima de $5 \mathrm{mg} / \mathrm{L}$. Por esse motivo, a concentração de nitrogênio amoniacal é importante parâmetro de classificação das águas naturais e é normalmente utilizado na constituição de índices de qualidade das águas. Os nitratos são tóxicos e causam uma doença chamada metahemoglobinemia infantil, que é letal para crianças (o nitrato se reduz a nitrito na corrente sangüínea, competindo com o oxigênio livre, tornando o sangue azul). Por isso, o nitrato é padrão de potabilidade, sendo $10 \mathrm{mg} / \mathrm{L}$ o valor máximo permitido pela Portaria MS 1469. Todas as amostras coletas apresentaram resultados se mostraram dentro dos padrões neste parâmetro (Tabela 1).

Tabela 1. Resultados das análises de amostras coletadas realizadas em 08/06/2004

\begin{tabular}{|c|c|c|c|c|c|c|c|c|c|c|c|c|}
\hline PARÂMETROS & Unidades & $\begin{array}{l}\text { Poço } 1 \\
\text { Result. }\end{array}$ & $\begin{array}{l}\text { Poço } 2 \\
\text { Result. }\end{array}$ & $\begin{array}{l}\text { Poço } 3 \\
\text { Result. }\end{array}$ & $\begin{array}{l}\text { Poço } 4 \\
\text { Result. }\end{array}$ & $\begin{array}{l}\text { Poço } 5 \\
\text { Result. }\end{array}$ & $\begin{array}{l}\text { Poço } 7 \\
\text { Result. }\end{array}$ & $\begin{array}{l}\text { Poço } 8 \\
\text { Result. }\end{array}$ & $\begin{array}{l}\text { Poço } 10 \\
\text { Result. }\end{array}$ & $\begin{array}{l}\text { Poço } 11 \\
\text { Result. }\end{array}$ & $\begin{array}{c}\text { Poço } 12 \\
\text { Result. }\end{array}$ & $\begin{array}{c}\text { Rio } \\
\text { Result. }\end{array}$ \\
\hline N Total (NKT) ${ }^{(\mathrm{a})}$ & $\mathrm{mg} \mathrm{N} / \mathrm{L}$ & 850 & 8,96 & 49,3 & 0 & 11,2 & 5,17 & 5,17 & 0 & 5,17 & 1,7 & 6,9 \\
\hline N Amoniacal ${ }^{(\mathrm{b})}$ & $\mathrm{mg} \mathrm{N} / \mathrm{L}$ & 0,234 & 0,073 & 0,076 & 0,074 & 0,075 & 0,079 & 0,158 & 0,182 & 0,154 & 0,158 & 0,132 \\
\hline
\end{tabular}

(a) Nitrogênio Kjeldahl Total

(b) Nitrogênio Amoniacal - Método Voltamétrico

\section{Conclusão}

Os resultados demonstraram que o filme de polipirrol dopado com DBSA depositado sobre carbono vítreo possui o melhor desempenho para determinação de amônia.

$\mathrm{O}$ aumento da concentração de íons amônio provoca um aumento na corrente de pico anódico. A análise da corrente de pico em função da concentração de íons amônio leva a um comportamento linear com uma sensibilidade de igual a 4,2 mA mM${ }^{-1} \mathrm{~cm}^{-2}$, em uma faixa de concentração de 0 e $80 \mathrm{mM}$.

\section{Referências}

BLANC, J.P.; DEROUICHE, N.; EL HADRI, A.; GERMAIN, J. P.; MALEYSSON, C.; ROBERT, H. Study of the action of gases on a polypyrrole film. Sensors and Actuators B, Lausanne, v.1, n.1/6, p.130-133, jan. 1990.

DALL'ANTONIA, L. H.; MIYATA, M. E. V.; CORDOBA DE TORRESI, S. I.; TORRESI, R. M. A new sensor for ammonia for determination based on polypyrrole films doped with dodecilbenzenesulfonate (DBSA) ions. Electroanalysis, New York, v.14, n.22, p.1577-1586, nov. 2002.
GUSTAFSSON, G.;LUNDSTRÖM, I.;LIEDBERG, B.; WU, C. R.; INGANAS, O.; WENNERSTROM, O. The interaction between ammonia and poly(pyrrole). Synthetic Metals, Lausanne, v.31, n.2, p.163-179, aug. 1989.

GUSTAFSSON, G.; LUNDSTRÖM, I. The effect of ammonia on the physical properties of polypyrrole Synthetic Metals, Lausanne, v.21, n.2, p.203-208, sep. 1987.

HEIDUSCHKA, P.; PRESCHEL, M.; RÖSCH, M.; GÖPEL, W. Regeneration of an electropolymerised polypyrrole layer for the amperometric detection of ammonia. Biosensors \& Bioelectronics, Essex,., v.12, n.12, p.12271231, dec. 1997.

KANAZAWA, K. K.; DIAZ, A. F.; GEISS, R. H.; GILL, W. D.; KWAK, J. F.; LOGAN, J. A.; RABOLT, J. F.; STREET, G. B. 'Organic metals': polypyrrole, a stable synthetic 'metallic' polymer. Journal of the Chemical SocietyChemical Communications, London, v.19, p.854-855, 1979.

KRUTOVERTSEV, S. A.; SOROKIN, S. I.;ZORIN, A. V.; LETUCHY, Y. A.; ANTONOVA, O. Y. Polymer film-based sensors for ammonia detection. Sensors and Actuators $B$ , Chemical, Lausanne, v.7, n.1/3, p.492-494, mar. 1992.

LÄHDESMÄKI, I.; KUBIAK, W. W.; LEWENSTAM, A.; IVASKA, A. Interferences in a polypyrrole-based amperometric ammonia sensor. Talanta, London, v.52, n.2, p.269-275, jun. 2000.

LUBENTSOV, B.Z.; TIMOFEEVA, O. N.; KHIDEKEL, M. L. Conducting polymer interaction with gaseous

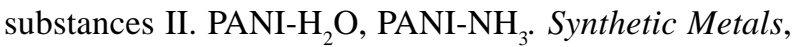
Lausanne, v.45, n.2, p.235-240, nov. 1991. 
MIASIK, J. J.; HOPER, A.; MOSOLEY, P. T.; TOFIELD, B. C. In: . Conducting Polymers. Reidel, Dordrecht: L. Alcacer, 1987. p.189.

MIASIK, J. J.; HOPER, A.; TOFIELD, B. Conducting polymer gas sensors. Journal of the Chemical Society, London, v.82, p.1117-1126, 1986.

NYLANDER, C.; ARMGARTH, M.; LUNDSTRÖM, I. An ammonia detector based on a conducting polymer. Anal. Chem. Symp. Ser., v.17, p.203-207, 1983.

RATCLIFFE, N. M. Polypyrrole-based sensor for hydrazine and ammonia. Analytica Chimica Acta, Amsterdam,v.239, n.2, p.257-262, dec. 1990.

SLATER, J.; WATT, E. J.; FREEMAN, N. J; MAY, I. P.; WEIR, D. J. Gas and vapour detectionwith poly (pyrrole) gas sensors. Analyst, London, v.117, n.8, p.1265-1270, aug. 1992

TORRESI, R. M.; CORDOBA DE TORRESI, S. I.; MATENCIO, T.; DE PAOLI, M. A. Ionic exchanges in dodecylbenzenesulfonate-doped polypyrrole Part II: Electrochemical quartz crystal microbalance study. Synthetic Metals, Lausanne, v.72, n.3, p.283-287, jun. 1995.

TROJANOWICZ, M.; LEWENSTAM, A.; KRAWCZYK, T. K. V.; LÄHDESMÄKI, I.; SZCEPEK, W. Flow injection amperometric detection of ammonia using polypyrrolemodified electrode and its application in urea and creatinine biosensors. Electroanalysis, New York, v.8, n.3, p.233243, mar. 1996.
VIDOTTI, M.; DALL'ANTONIA, L. H.; BERGAMASKI, K.; NART, F. C.; CORDOBA DE TORRESI, S. I. "'On line" mass spectrometric detection of ammonia oxidation products generated by polypyrrole based amperometric sensors. Analytica Chimica Acta, Amsterdam, v.489, n.2, p.207-214, aug. 2003.

VIDOTTI, M.; DALL'ANTONIA, L. H.; CINTRA, E. P.; CORDOBA DE TORRESI, S. I. Reduction of interference signal in poly(pyrrole) based ammonia sensors. Electrochimica Acta, New York, v.49, n.22/23, p.3665-3670, sep. 2004.

VIGMOND, S. J.; KALLURY, K. M. R.; GHAEMMAGHAMI, V.; THOMPSON, M. Characterization of the polypyrrole film-piezoelectric sensor combination. Talanta, London, v.39, n.4, p.449456, apr. 1992.

WNEK, G. E.. A proposal for the mechanism of conduction in polyaniline. Synthetic Metals, Lausanne, v.15, n.2/3, p.213-218, jul./aug. 1986.

WYERS, G. P.; OTJES, R. P.; SLAMINA, J. A continuous,flow denuder for the measurement of ambient concentrations and surface-exchange fluxes of ammonia. Atmospheric Environment Part a - General Topics, Oxford, v.27, n.13, p.2085-2090, sep. 1993. 